\title{
Neuralgias of the trigeminal nerve
}

\author{
Allan S Gordon MD FRCPC
}

\begin{abstract}
AS Gordon.
Neuralgias of the trigeminal nerve.

Pain Res Manage 2000;5(1):107-113.
\end{abstract}

Practitioners are often presented with patients who complain bitterly of facial pain. The trigeminal nerve is involved in four conditions that are sometimes mixed up. The four conditions trigeminal neuralgia, trigeminal neuropathic pain, postherpetic neuralgia and atypical facial pain - are discussed under the headings of clinical features, differential diagnosis, cause and treatment. This article should help practitioners to differentiate one from the other and to manage their care.

Key Words: Atypical facial pain; Facial pain; Postherpetic neuralgia; Trigeminal neuralgia; Trigeminal neuropathic pain

\section{Les névralgies du nerf trigéminé}

RÉSUMÉ : Les médecins voient souvent dans leur pratique, des patients ennuyés par une intense douleur au visage. Le nerf trigéminé est en cause dans quatre maladies que l'on confond parfois. On aborde ici ces quatre maladies, soit la névralgie trigéminée, la douleur neuropathique trigéminée, la névralgie post-zostérienne et la douleur faciale atypique, sous les rubriques suivantes : caractéristiques cliniques, diagnostic différentiel, cause et traitement. Cet article devrait aider les médecins à les distinguer les unes des autres et à les traiter adéquatement.
$\mathrm{P}$ ractitioners are often presented with patients who complain bitterly of pain in the face. Once temporomandibular joint disease, oral and dental disease, sinus disease and ocular disease have been excluded, the health care practitioner is left with several conditions that must be considered and often require an active differential diagnosis. These conditions include trigeminal neuralgia (TN), trigeminal neuropathic pain (TNP), postherpetic neuralgia (PHN) and atypical facial pain (AFP).

Cluster headache, facial migraine and temporal arteritis may also cause facial pain but are beyond the scope of this review. Burning mouth syndrome, probably a variant of AFP, is also not considered.

All four conditions can produce quite severe pain, and the distribution of the pain is through the territory of the trigeminal nerve, hence the designation 'neuralgias of the trigeminal nerve'.

The present article describes each condition under the fol- lowing headings: clinical features, differential diagnosis, cause, treatment and evaluation.

\section{EVALUATION}

The most important tools for evaluating neuralgias of the trigeminal nerve are the history and physical examination, and, given enough time and motivation, most practitioners may suspect the diagnosis. However, a multidisciplinary approach is often necessary to include neurology, dentistry and at times otolaryngology and ophthalmology. At the Headache and Facial Pain Unit of the Wasser Pain Management Centre, Toronto, Ontario, even the most 'transdisciplinary' of physicians feel the need to consult with colleagues to be certain in many of these difficult cases.

ANATOMY OF THE TRIGEMINAL NERVE

The trigeminal nerve is composed of three large branches: the ophthalmic (V1, sensory), maxillary (V2, sensory) and man-

\footnotetext{
Wasser Pain Management Centre, Mount Sinai Hospital, and Departments of Medicine and Neurology, University of Toronto, Toronto, Ontario Correspondence and reprints: Dr Allan S Gordon, 600 University Avenue \#1170, Toronto, Ontario M5G 1X5. Telephone 416-586-5181, 
dibular (V3, motor and sensory) branches. The large sensory root and smaller motor root leave the brainstem at the lateral surface of the pons. The sensory root terminates in the largest of the cranial nerve nuclei, which extends from the pons all the way down into the second cervical level of the spinal cord. The sensory root joins the trigeminal ganglion between the layers of the dura mater in a depression on the floor of the middle cranial fossa (Meckle's cave). The motor root originates from cells located in the motor nucleus of the trigeminal nerve located in the midpons of the brainstem. This root passes through the trigeminal ganglion and combines with the corresponding sensory root to become the mandibular nerve. It is distributed to the muscles of mastication, the mylohyoid muscle, the anterior belly of the digastric, and the tensor veli palatini and tensor tympani muscles. The three sensory branches of the trigeminal nerve branch out from the ganglion. The ophthalmic and maxillary branches travel in the wall of the cavernous sinus and then leave the skull. The ophthalmic branch travels through the superior orbital fissure, through the orbit to reach the skin of the forehead and top of the head. The maxillary nerve enters the cranium through the foramen rotundum via the pterygopalatine fossa. Its sensory branches reach the pterygopalatine fossa via the inferior orbital fissure (face, cheek and upper teeth) and pterygopalatine canal (soft and hard palate, nasal cavity and pharynx). There are also meningeal sensory branches that enter the trigeminal ganglion within the cranium. The sensory part of the mandibular nerve is composed of branches that carry general sensory information from the mucous membranes of the mouth and cheek, anterior two-thirds of the tongue, lower teeth, skin of the lower jaw, side of the head and scalp, and meninges of the anterior and middle cranial fossae.

Cranial nerve $\mathrm{V}$ is served by several nuclei in the brainstem and upper cervical cord. These nuclei include the sensory nucleus of $\mathrm{V}$, motor nucleus of $\mathrm{V}$, spinal nucleus and tract of V, and the mesencephalic nucleus of V. All of these except the motor nucleus are sensory. The pain and temperature fibres descend to the spinal nucleus topographically where they synapse with the second order neurons. These neurons ascend to the thalamus where they synapse. Some fibres may go to the medial thalamus. The third order neuron then climbs to the somatosensory cortex and also the insula. However, many functional studies suggest rich connections with other centres, including the anterior cingulate, and others suggest that these connections are beyond the scope of review but are likely important in central pain mechanisms.

\section{SPECIFIC SYNDROMES}

TN

Clinical features: There are few human conditions more tormenting than the sharp, severe, jabbing pains of TN or tic doloureux. Long known as a severely painful condition affecting an older population, patients are subject to numerous attempts at surgical and medical treatment yet are frequently misdiagnosed.

Primary TN commonly manifests after the age of 50 years, and although it can present in the third or fourth decade, these cases are notable. An early onset should always suggest the possibility of a secondary TN; therefore, another diagnosis should be sought. Females are usually cited as being affected slightly more often than males, three to two, although some studies suggest that the incidence may be equal. An attack is classically unilateral, with the right side being more commonly affected than the left. The condition clearly respects anatomical boundaries, affecting one or more branches of the fifth cranial nerve (V). The third (mandibular) division, or $\mathrm{V} 3$, is most commonly involved, with the second (maxillary), or V2, and the first (ophthalmic), or V1, following, respectively. Simultaneous involvement of V2 and $\mathrm{V} 3$ is also quite common. The condition is never simultaneously bilateral, but $3 \%$ of patients are affected on both sides during different attacks.

The pain is electric-like or shock-like, of short duration and spasmodic, generally causing the patient to wince or eliciting an avoidance reaction. At times, a series of lancinating pains come together in a staccato-like manner. The attacks can be stereotyped. There may be pain-free periods between attacks and occasionally months or years can intervene. Trigger spots are common in the scalp, nostril, cheek, face, upper lip, teeth or gums. The patient usually says that even lightly touching these areas, such as with a hairbrush, a wisp of cotton, a jet of cold air, a washcloth, a toothbrush or a razor, triggers the pain. Talking or kissing can induce a spasm of pain. Some patients refuse to wash or shave the affected part of their face or brush their teeth. Presentation in an older person with poor dentition may lead to the mistaken diagnosis of dental disease, resulting in unnecessary dental treatment or extraction. Some have a constant pain prodrome at the outset, and that may complicate the early diagnosis. Sometimes patients have a constant, burning, background with superimposed tic-like bursts.

Increasingly, there have been reports of variants, including a cluster-tic syndrome with features of cluster headache and TN. Atypical TN is also reported - a variation not included in the classifications to date. Most atypical TN would be classified as TNP or secondary TN. Some prefer to call atypical $\mathrm{TN}$ a chronic, more serious form of $\mathrm{TN}$.

\section{Differential diagnosis}

When there is an accompanying sensory alteration or other cranial nerve abnormality, a secondary cause should be sought. Schwannomas and meningiomas affecting the nerve, and multiple sclerosis affecting the brainstem should be considered. A recent study of approximately 3000 patients showed that $10 \%$ had a tumour. The author recalls a case of carcinoma of the maxillary sinus presenting as TN-like spasmodic facial pain. Quite often, structural lesions present with trigeminal neuropathic pain, which is more constant, is often associated with sensory loss and does not share all the spasmodic features of TN. These lesions can include trauma, sometimes surgical trauma, to branches of the trigeminal nerve.

Dental disease may mimic TN, and TN may present as 
dental pain. In dental presentations, pulpitis, 'cracked' roots and periodontal disease need to be excluded. Rarely, cases are seen that begin after dental trauma. AFP can usually be differentiated by the characteristics of the pain; AFP is not spasmodic and is more constant. When chewing induces pain, disorders of the TM joint apparatus must be considered. When the pain affects V3, dental and temporomandibular joint (TMJ) disease must be considered. In V2 pain, dental and sinus disease is to be ruled out. Sometimes chewing induces the pain. In those circumstances, a TMJ disturbance also needs to be considered.

Careful neurological examination is mandatory to exclude sensory loss or other alterations suggestive of a primary lesion or disease. Similarly, careful dental and sinus examinations are also necessary, including enlisting the aid of imaging techniques. If a secondary cause is suspected or if the patient is young, appropriate magnetic resonance imaging is essential.

\section{Cause}

Jannetta $(1,2)$ has suggested that TN results from compression of the trigeminal nerve root by an adjacent artery or other vascular lesion with continuous pulsation leading to secondary changes and spontaneous discharges. All of Broggi et al's (3) recent 250 cases had microvascular compression at surgery. The superior cerebellar artery may compress the rostrum component of the nerve, producing V2 and V3 pain. The anterior inferior cerebellar artery may compress the caudal and posterior portion of the nerve producing V1 pain. Basil artery ectasia is associated with TN. Burchiel and Slavin (4) recently opined that typical TN evolves into atypical TN and then TNP based on the degree of compression and damage, with increasing sensory findings along the way.

Some authors have popularized the concept of a peripheral cause of TN, with jaw microinfections leading to localized nerve damage and deafferentation. Adams et al (5) popularized the concept of neuralgia-inducing cavitational necrosis. Another concept also not proven is that a peripheral irritant, such as a TMJ problem, causes a 'sensitized neural system'.

Central theories suggest hyperactivity in the trigeminal nucleus and reverberating circuits centrally, as with epilepsy. Pagni (6) presented a unifying theory that suggested that TN is a type of sensory reflex epilepsy. The posterior root is damaged or distorted by a vessel, or compressed by another lesion, producing demyelination and/or microneuromas. This chronic damage leads to ectopic impulses and afterdischarges that kindle spontaneous impulse generation in the gasserian ganglion and an epileptic subliminal focus in the trigeminal nucleus that induces 'seizures' of pain in the gasserian ganglion and the trigeminal nucleus.

\section{Treatment}

The mainstay of medical treatment of TN is carbamazepine, which controls symptoms in $70 \%$ to $80 \%$ of those treated. If there is no initial response to this drug, the diagnosis should be reconsidered. Occasionally, however, a nonspecific response to carbamazepine may occur in other conditions, so carbamazepine responsiveness should not be the definitive diagnostic criteria. Lithium and carbamazepine have been used to treat tic cluster. The use of topical capsaicin intraorally has also been advocated. When carbamazepine is used, monitoring of liver function is mandatory. There have been reports of $30 \%$ to $35 \%$ success rates with diphenylhydantoin. Baclofen is helpful at times, as is valproate and pimozide. In refractory cases, combinations of drugs may have to be enlisted. There have been some studies using gabapentin, but these are still uncontrolled or case studies. There is a clinical impression that gabapentin is effective in escalating doses (up to $3600 \mathrm{mg} /$ day or more). Opiates, nonsteroidal antiinflammatory drugs and analgesics are minimally effective in pain control.

Nerve blocks are sometimes used, as is peripheral avulsion, but most surgeons prefer a percutaneous radiofrequency gangliolysis. This works well, and the pain is relieved for quite some time. Unfortunately, anesthesia is invariable. Rarely, a particularly painful and difficult to manage combination of continuous pain and anesthesia in the affected nerve (anesthesia dolorosa) may ensue. This condition is mostly likely due to deafferentation and denervation hypersensitivity. Glycerol injection, which has fewer side effects, has been proposed but may not be as effective. Jannetta (2) popularized suboccipital craniotomy with microvascular decompression. He reported a decreasing number of complications as the techniques improved, calling it an extremely safe and effective treatment. Romansky et al (7) reported about $90 \%$ effectiveness in 85 patients, and it is advocated in elderly patients. Broggi et al (3) reported a $15.3 \%$ recurrence rate in his 250 patients. Gamma knife radiosurgery has many proponents. Loeser (8) suggested an $85 \%$ success rate, with a $0.5 \%$ mortality rate, using this therapeutic modality. Acupuncture is not particularly successful in treating these patients. Balloon compression of the gasserian ganglion has been successfully used; a recent study of 158 patients reported no deaths or serious complications (9).

\section{TNP}

\section{Clinical features}

Chronic TNP conditions have a number of unique properties, including being decidedly difficult to treat, and are in essence refractory. TNP has often been called 'atypical TN' or secondary TN. The patient has facial pain that is severe, burning or boring. It may be on the skin or within the mouth. Sometimes, touching the area lightly may induce allodynia (pain induced by mechanical touch) or hyperalgesia (excessive pain response to painful stimulus). As opposed to TN, there may be sensory loss as well. There may be a temporal relationship to trauma or some other illness, or it may be unexplained. The pain is constant rather than paroxysmal. A particularly disabling pain is produced by injury to the trigeminal nerve or its branches. This is not unexpected after severe facial trauma, 
but it is also common following surgical interventions. In our clinic, we have seen numerous cases of infraorbital nerve injuries after Caldwell-Luc or other sinus surgery, inferior alveolar nerve injury after lower third molar extraction and lower quadrant endodontic procedures, or maxillary branch injuries with upper quadrant dental procedures These patients have anatomically correct hypesthesia, hyperesthesia, allodynia and severe pain, often defying conservative management.

\section{Differential diagnosis}

The lesion may be peripheral or central. Multiple sclerosis is the classical central cause of this syndrome, although it has been reported with stroke and tumour along the trigeminal pathway. Most cases are of peripheral cause. In addition to dental or facial trauma in our clinic, vasculitis, collagen vascular disorder, TMJ surgery and 'idiopathic' are the most common causes seen (unpublished data). Surgical procedures are sometimes done on inferior alveolar nerve injuries, but those do not necessarily prevent the development of pain. Generally, treatment includes drug therapy (tricyclics, antiepileptics) or analgesics. Various neurostimulatory techniques can also be attempted. Acupuncture treatment is often very difficult, with a poor response rate.

Secondary breast cancer to the skull is another possibility. Burchiel (10) reported a large series of patients, emphasizing sensory loss, deafferentation and nontic-like pains. Benoliel and Sharav (11) wrote a review entitled "Neuropathic orofacial pain", which also described the entity. Some authors reason that some idiopathic cases may also relate to microvascular compression and that the reason these cases do not present as TN is a matter of degree of compression.

\section{Cause}

The pathophysiology is usually damage to a branch on the trigeminal nerve. Instead of causing mere numbness, the injury produces a neuropathic pain state. There is peripheral injury that most likely causes peripheral and then central sensitization with neuroplastic changes. The etiology of the pain relates to the complications of nerve injury, including deafferentation, neurogenic inflammation and sympathetic hyperactivity.

\section{Treatment}

Poor treatment response is characteristic. Sometimes, opiates such as long acting oxycodone, slow release morphine or a fentanyl patch are used, but there are no good randomized controlled trials or clinical trials that confirm their effects on this specific pain condition. Tricyclics and valproic acid may be of some benefit. Randomized, controlled trials of gabapentin, topiramate, tramadol and some of the upcoming new selective sodium channel blockers are necessary to determine their effectiveness and efficacy. The role of acupuncture is unknown. Treatment is generally difficult. There are various surgical approaches to the treatment of traumatic nerve lesions, including removal of neuromas and nerve grafts on inferior alveolar lingual nerve lesions, but these do not necessarily prevent the development of pain.

\section{ACUTE HERPES ZOSTER AND PHN Clinical features}

The varicella zoster virus may produce two very painful neuropathic pain syndromes: the acute herpes zoster skin and mucosal lesions known as shingles, and the difficult, chronic pain condition known as PHN.

Herpes zoster, or shingles, is a reactivation of the varicella zoster virus, usually contracted as chickenpox during childhood. The virus appears to lay dormant in the trigeminal, geniculate and dorsal root ganglia for many years, and for various reasons becomes activated later in life. This can occur in immunocompromised individuals (eg, those suffering from acquired immunodeficiency syndrome or lymphoma), or it may occur in the elderly with no known immunological disturbance but in whom cell-mediated immunity may have lessened. When reactivation occurs, the virus migrates down the sensory nerve fibres, producing skin and/or mucosal vesicles in a dermatomal distribution.

In $10 \%$ to $15 \%$ of people affected by acute herpes zoster, the trigeminal nerve is involved. Eighty per cent of these involve the first division of the trigeminal nerve, involving the forehead and the eye (ophthalmic herpes zoster). This can involve the extraocular nerves as well. The other common presentation is known as the Ramsey Hunt syndrome, in which facial palsy is associated with vesicles involving the ear, the external canal, and sometimes the skin inferior or anterior to the canal (fifth cranial nerve) and the eighth cranial nerve, producing vertigo and auditory symptoms. In this syndrome, the virus is postulated to lay dormant in the geniculate ganglion.

Pre-eruptive pain is common up to two to three days before the blisters appear, and may last up to three weeks. The acute phase is very painful in the area involved, with a constant burning, gnawing pain, superimposed by a burst of shock-like, brief, tic-like pains. These are distributed through the dermatome. There may be areas of anesthesia and hyperesthesia around the vesicles. In most cases, after a few weeks, the vesicles heal, leaving scars, and the pain disappears. In a small percentage, $9 \%$ to $14 \%$, another kind of pain may develop subsequently. This is known as PHN. It has been suggested that $52 \%$ of patients with herpes zoster ophthalmicus have PHN, with $22 \%$ presenting by one year (12).

Most authors do not define the persisting pain as PHN unless it lasts for three to six months. In their classical study, Watson et al (13) noted that 47 of 208 patients with PHN had trigeminal involvement. Sixty-five per cent of the group were women, but it was concluded that this reflected the usual predominance of women alive in the older age group. Fifty per cent of patients with herpes zoster at age 60 years and $75 \%$ of patients at age 70 years developed PHN at one month after the rash. Watson et al (14) stated that $5 \%$ of patients with all types of zoster have pain at three months and that $3 \%$ have severe pain at one year. Cobo et al's (12) observations suggest that the trigeminal nerve neuralgias may act differently from other neurological disturbances. A 1995 article by Watson (15) stated that, at age 60 years, about $50 \%$ of patients with herpes zoster suffer significant pain. 
Postherpetic pain is similar to the acute symptom, with a constant, severe background pain and superimposed tic-like spasms. There may be other sensations as well. Watson et al (16) described hyperesthesia, dysesthesia or allodynia in the areas involved. The scars may be anesthetic, but there may be persisting areas of hypoesthesia or hyperesthesia inside and outside the area of scarring. This commonly occurs in the forehead or around the ear in cranial PHN. The pain may be described as burning, gnawing, stabbing, shooting and sharp. Physical activity and emotional factors may exacerbate the pain. This can be incapacitating, particularly in an older patient.

There are three types of results - pain such as allodynia; abnormal sensations such as anesthesia, paresthesia, dysesthesia, prickling, itching and burning; and complete recovery. This may be an important guide to pathophysiology and deciding on treatment. Thus, the symptoms may result from different fibres being affected or destroyed.

\section{Differential diagnosis}

Usually, differential diagnosis is not an issue. There is usually a history of acute rash followed by neuralgic pain. There are scars and areas of allodynia, hypesthesia and normal sensation. Immune deficiency must be excluded. The pain can usually be easily differentiated from TN and TNP by the nature of the history.

\section{Cause}

The acute pain is caused by the virus invading the ganglion, nerve root and nerve, producing a hemorrhagic inflammation. Rarely, leptomeningeal and perenchymal involvement can occur. Later, with progression of the disease, fibrosis can be found in the ganglion, nerve root and nerve. Some pathological studies have demonstrated dorsal column involvement lasting up to nine months. The persisting pain may be associated with a relative loss of peripheral large fibres, with persistence of smaller substance P-containing smaller fibres. Central deafferentation is said to be responsible for much of the persisting pain, but peripheral factors are likely important as well. There is recent evidence of heat hyperalgesia and an increase in C-nociceptor activation $(17,18)$. The acute pain is caused by the virus invading the ganglion, nerve root and nerve.

\section{Treatment}

Acyclovir is said to lessen the time course of acute herpes zoster but not to lessen the appearance of PHN. Bilora et al (19) found that younger patients fared better with acyclovir. Huff et al $(20,21)$ suggested a decreased incidence of PHN with acyclovir, and Hoang-Xuan et al (22), in a series of patients with ophthalmic herpes, found fewer complications and more rapid healing with acyclovir. Famcyclovir is being used more frequently as is pencyclovir and valacyclovir (23). There is no benefit of prescribing prednisone specifically for the prevention of PHN (24). Hopefully, the advent of the varicella vaccine will significantly decrease the incidence of both chickenpox and shingles (25).
In 1995, Watson (15) reported that it was possible to obtain relief of pain in $60 \%$ to $70 \%$ of patients with PHN. Until recently, the mainstay of treatment has been the tricyclics, particularly amitriptyline, in doses averaging $75 \mathrm{mg} /$ day, although side effects (dizziness, dryness of mouth and weight gain) and, in older people, cardiological effects, are of concern. A randomized, double-blind, placebo controlled parallel design study reported that gabapentin $3600 \mathrm{mg}$ had a $30 \%$ advantage over placebo, helping both pain and sleep. Tramadol has also been shown to be effective in an open label study compared with clomipramine with or without levomepromazine. Chlorprothixene may be effective but only in doses higher than $100 \mathrm{mg}$. Other suggested treatment approaches have recently been reviewed by Rowbotham et al (26). Topical capsaicin applied locally can reduce the pain, although its use is awkward and patients object to the burning sensation, particularly around the eyes. Nerve blocks, topical acetylsalicylic acid, acupuncture, transcutaneous electrical nerve stimulation, topical local anesthetic and lidocaine/prilocain cream have been tried with some success. Sympathetic blockade has also been advocated. Fortunately. the symptoms gradually lessen with time. Because there is central deafferentation, producing more distal lesions is ineffective; therefore, permanent nerve block, and most of the surgical procedures used to treat TN, should not be used. Opioids have classically been thought to be ineffective for neuropathic pain. However, Watson and Babul (27) demonstrated the effectiveness of long acting oxycodone in relieving the pain of PHN. Filadora et al (28) treated three patients with acute shingles and two with PHN with excellent results. All patients reported complete pain relief after titration with gabapentin up to $1800 \mathrm{mg} /$ day. The patients noted a dosedependent decrease in pain almost immediately after starting gabapentin. Specifically, a reduction in the frequency and intensity of allodynia, burning pain, shooting pain and throbbing pain was noted. Rowbotham et al (26) reported a multicentred, randomized, double-blinded, placebo controlled, parallel design study with 229 patients. None of the patients experienced side effects from the drug.

\section{AFP}

\section{Clinical features}

Frazier and Russell (29) first described AFP in 1924, differentiating it from TN. It falls within the category of 'facial pain not fulfilling other criteria' in the classification system of the International Headache Society (classification 12.8). As this classification implies, the diagnosis is generally one of exclusion, that is, it is only made after local orofacial disease, neurological disorders and related systemic diseases have been ruled out. It is not associated with objective neurological, facial or oral findings (as opposed to TNP), and often presents with a nonanatomical and even migratory pattern. Understanding the condition is complicated further by a plethora of other terms, used synonymously or as variations on the theme. These include phantom tooth pain, atypical odontalgia, atypical facial neuralgia, migratory odontalgia, the wandering tooth syndrome and dental causalgia. Because 
it is based on diagnosis by exclusion and is poorly understood, this condition may be missed or overdiagnosed and thus, lends itself to abuse.

AFP (30) is more common in women with an average age between 40 and 50 years but can occur from age 20 to 70 years, with a bimodal distribution $(31,32)$. The patient generally has a constant, burning or boring deep pain that is poorly defined. This contrasts with TN, where the pain is well localized, lancinating and paroxysmal, with a defined trigger zone or site. Patients often present with symptoms suggestive of dental etiology. The pain may be limited to one side of the face but can be unanatomical in distribution, even crossing the midline, and may involve multiple oral quadrants, either simultaneously or sequentially. The patients generally have a distinct presentation. They are often angry about their treatment history (not necessarily without justification) and have a peculiar, often exaggerated, response to placebos or active drugs (eg, tricyclics or calcitonin) $(33,34)$. They generally have little insight into their problem, demand solutions and blame previous practitioners. The patient often arrives with lengthy, detailed chronicles of their symptoms and treatment, a personality trait that actually helps diagnosis and treatment because it often documents the previous treatments very well.

\section{Differential diagnosis}

Dental disease is often suspected and indeed must be ruled out first, but this can also result in extensive, usually unnecessary dental treatment, particularly endodontic therapy and extractions. Unfortunately, inappropriate dental treatment can perpetuate or even aggravate the patient's pain. In some cases, the pain is precipitated historically by a dental or surgical procedure, or facial trauma, often quite minor. However, although a neuropathic etiology may be suspected, it often cannot be proven because of a lack of sensory findings. When the patient's pain has a throbbing character, a migrainous or vascular basis may be suspected. Similarly, if the cervicofacial musculature is tender, myofascial pain may be diagnosed. There are often associated, possibly predisposing and/or perpetuating affective disorders, somatization, personality disturbances or sleep disorders.

The condition can usually be differentiated from the other trigeminal conditions by the clinical features or lack thereof.

\section{Causes of AFP}

Although various theories have been presented, the etiology of AFP is uncertain. An undefined patient-specific susceptibility factor, predisposing this population to a chronic pain syndrome, appears to be present. Some have suggested deafferentation, resulting in central neuronal hyperactivity, in cases where nerve injury or trauma precipitated the symptoms $(35,36)$.

Loeser (8) described four types of unilateral AFP. The first is associated with sensory findings and nerve damage after sinus or dental surgery, trauma or surgery for TN. One must question whether patients with this type of unilateral syndrome should be included in this diagnostic category in light of the presence of detectable neurological change; a diagnosis of TNP is more appropriate. The second type of unilateral AFP progresses insidiously, ultimately demonstrating clear evidence of TN. An organic basis, such as neoplasia or infection, is ultimately demonstrated in these cases; therefore, they are best classified as a secondary TN or as TNP rather than as AFP. The third type of unilateral AFP is associated with vascular features. It should be classified as migrainous and be labelled 'vascular orofacial migraine' (37). Finally, the fourth type occurs without a particular precipitating event, and the patient has a burning, constant, usually circumscribed pain. This classification does not relate the dental or other precipitating or exacerbating factors to the condition. Other objective parameters need to be delineated to classify adequately and, more important, to understand these conditions. This last type is the one that most closely fits the classical definition of AFP.

It is likely that AFP is not a single clinical entity but rather a number of disorders, each with different etiological factors. Some may be true neuropathic pains, related to a peripheral nerve injury that cannot be documented or inappropriate activity within the sympathetic nervous system, such as has been suggested elsewhere in the body, and others may result from a central nervous system disorder.

One unique clinical presentation of AFP, often referred to as atypical odontalgia or phantom tooth pain, presents as a toothache. The patient can localize the area, even the tooth assumed to be the cause of the pain. A vascular or neurovascular etiology has been suggested.

A more prevalent theory of AFP is that the symptoms are related to a psychological disorder. For example, a large proportion of these patients have been shown to suffer from clinical depression; however, depression is a common finding in most populations of chronically ill patients and whether this is a cause or effect remains to be demonstrated and is discussed further below. A high percentage of patients with histories of physical and sexual abuse in a facial pain clinic population has been found, and it has been suggested that somatization is an important mechanism in chronic facial pain, a factor suggested by their profoundly adverse responses to calcitonin, which generally produces mild and transient side effects in other populations. Thus, psychogenic factors are important in AFP, perhaps by opening or closing various 'gates', either peripherally or centrally. Our own belief is that, although 'psychological' aspects are important, these patients need to be treated as having a 'real' disease.

\section{Treatment of AFP}

Patients are generally not compliant with prescribed treatment. Tricyclic amines may offer relief, but patients may discontinue the medication on their own prematurely or fail to follow prescribed directions. Gabapentin or topiramate may ultimately prove to be effective, but randomized, controlled clinical trials showing this are lacking. Tramadol is another likely candidate. Analgesics and nonsteroidal anti-inflammatory drugs are either ineffective or, at best, give temporary relief. It is important to avoid strong opiates. Neurosurgical 
procedures, including nerve blocks, are usually ineffective and counterproductive. Often, the clinician's best contribution to the patient's well being is preventing needless and often irreversible or invasive treatment. A multidisciplinary approach is necessary.

\section{CONCLUSIONS}

TN, TNP, PHN and AFP all produce their symptoms through the involvement of the trigeminal nerve. Clinicians must be able to differentiate these conditions from each other and from disturbances of the teeth, TMJ, eyes, nose, throat and sinuses. This article has defined these four disorders and has elucidated their causes and treatments. A multidisciplinary approach is necessary to diagnose and manage them effectively.

\section{REFERENCES}

1. Jannetta PJ. Arterial compression of the trigeminal nerve at the pons in patients with trigeminal neuralgia. J Neurosurg 1967;26:159-62.

2. Jannetta PJ. Outcome after microvascular decompression for typical trigeminal neuralgia, hemifacial spasm, tinnitus, disabling positional vertigo, and glossopharyngeal neuralgia. Clin Neurosurg 1977;44:331-83.

3. Broggi G, Ferrioli P, Franzini A, Servello D, Dones I. Microvascular decompression for trigeminal neuralgia: comments on a series of 250 cases, including 10 patients with multiple sclerosis. J Neurol Neurosurg Psychiatry 2000;68:59-64.

4. Burchiel KJ, Slavin KV. On the natural history of trigeminal neuralgia. Neurosurgery 2000;46:152-4.

5. Adams WR, Spolnik KJ, Bouquot JE. Maxillofacial osteonecrosis in a patient with multiple "idiopathic" facial pains. J Oral Pathol Med 1999;28:423-32.

6. Pagni CA. The origin of tic douloureux: a unified view. J Neurosurg Sci 1993;37:185-94.

7. Romansky K, Stoianchev N, Diney E, Iliev I. Results of treatment of trigeminal neuralgia by microvascular decompression of the (V)th nerve at its root entry zone. Arch Physiol Biochem 1998;106:392-6.

8. Loeser JD. Tic doloreux and atypical facial pain. In: Wall PD, Melzack R, eds. Textbook of Pain, 3rd edn. Edinburgh: Churchill Livingstone, 1994:699-710.

9. Correa CF, Teixera MJ. Balloon compression of the Gasserian ganglion for the treatment of trigeminal neuralgia. Stereotact Funct Neurosurg 1998;71:83-9.

10. Burchiel KJ. Trigeminal neuropathic pain. Acta Neurochir Suppl (Wien) 1993;58:145-9.

11. Benoliel R, Sharav Y. Neuropathic orofacial pain. Compend Contin Educ Dent 1998;11:1099-102.

12. Cobo M, Fowlkes GN, Liesegang T, et al. Observations on the natural history of herpes zoster ophthalmicus. Curr Eye Res 1987;6:195-9.

13. Watson PN, Morsehead C, Van Der Kooy D, et al. Postherpetic neuralgia postmortem. Pain 1988;341:29-38.

14. Watson CP, Evans RJ, Reed K, et al. Amitriptyline versus placebo in post herpetic neuralgia. Neurology 1982;32:670-3.

15. Watson CP. The treatment of postherpetic neuralgia. Neurology 1995;45(Suppl 8):S58-60.

16. Watson CP, Evans RJ, Watt VR. Post-herpetic neuralgia and topical capsaicin. Pain 1988;33:333-40.
17. Fields HL, Rowbotham M, Baron R. Postherpetic neuralgia: irritable nociceptors and deafferentation. Neurobiol Dis 1998;4:209-27.

18. Choi B, Rowbotham MC. Effect of adrenergic receptor activation on post-herpetic neuralgia pain and sensory disturbances. Pain 1997;69:55-63.

19. Bilora F, Genovese R, Presotto F, et al. Herpes zoster and post herpetic neuralgia. Comparison between elderly patients and young adults treated with acyclovir. Minerva Med 1994;85:333:896-900.

20. Huff JC, Beam B, Balfour HH Jr, et al. Treatment of herpes zoster with oral acyclovir. Am J Med 1988;85:89-94.

21. Huff JC, Drucker JL, Clemmen A, et al. Effect of oral acyclovir on pain resolution in herpes zoster: a reanalysis. J Med Virol 1993;1(Suppl):93-6.

22. Hoang-Xuan T, Buchi E, Herbort CP, et al. Oral acyclovir for herpes zoster ophthalmicus. Ophthalmology 1992;99:1062-70.

23. Snoeck R, Andrei G, De Clercq E. Current pharmacologic approaches to the therapy of varicella zoster virus infections: a guide to treatment. Drugs 1999;57:187-206.

24. Calza AM, Schmid E, Harms M. Systemic corticosteroids do not prevent post-herpetic neuralgia. Dermatology 1992;184:314-6.

25. Brunell P. Possible role of varicella vaccine in preventing herpes zoster. Pediatr Infect Dis J 1999;18:842-3.

26. Rowbotham M, Harden N, Stacey B, Bernstein P, Magnus-Miller L. Gabapentin for the treatment of postherpetic neuralgia: a randomized controlled trial. JAMA 1998;2;280:1837-42.

27. Watson CP, Babul N. Efficacy of oxycodone in neuropathic pain: a randomized trial in post-herpetic neuralgia. Neurology 1998;50:1837-41.

28. Filadora VA II, Sist TC, Lema MJ. Acute herpetic neuralgia and postherpetic neuralgia in the head and neck: response to gabapentin in 5 cases. Reg Anesth Pain Med 1999;24:170-4.

29. Frazier CH, Russell EC. Neuralgia of the face. An analysis of seven hundred and fifty four cases with relation to pain and other sensory phenomena before and after operation. Arch Neurol Psychiatry 1924;11:557-63.

30. Gordon AS, Mock D. Craniofacial pain. In: Irwin RS, Curley FJ, Grossman RF, eds. Diagnosis and Treatment of Symptoms of the Respiratory Tract. Armonk: Futura Publishing Company Inc, 1997.

31. Mock D, Frydman W, Gordon AS. Atypical facial pain: a retrospective study. Oral Surg Oral Med Oral Pathol 1985;59:472-4.

32. Sharav Y. Orofacial pain. In: Wall PD, Melzack R, eds. Textbook of Pain, 3rd edn. London: Churchill Livingstone, 1994:563-82.

33. Galli G, Gordon A, Shandling M, et al Diagnostic and prognostic features of atypical facial pain: A pilot study. J Orofacial Pain 1993;7:116. (Abst)

34. Schwartz G, Galonski M, Gordon A, et al. Effects of salmon calcitonin on patients with atypical facial pain: A randomized trial. J Orofacial Pain 1996;10:306-15.

35. Sessle BJ. Dental deafferentation can lead to the development of chronic pain. In: Klineberg I, Sessle BJ, eds. Orofacioal Pain and Neuromuscular Dysfunction: Mechanisms and Clinical Correlates. Oxford: Pergamon, 1985:115-29.

36. Sessle BJ. Neurobiology of facial and dental pain. In: Sarnat BC, Laskin DM, eds. The Temporomandibular Joint: A Biological Basis for Clinical Practice, 4th edn. Philadelphia: WB Saunders, 1992: 124-42.

37. Benoliel R, Elishoov H, Sharav Y. Orofacial pain with vascular type features. Oral Surg Oral Med Oral Pathol Oral Radiol Endod 1997;84;506-12. 


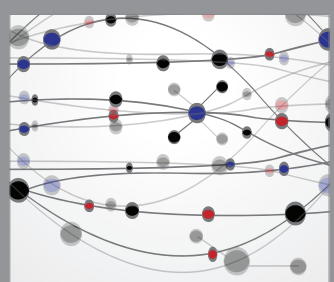

The Scientific World Journal
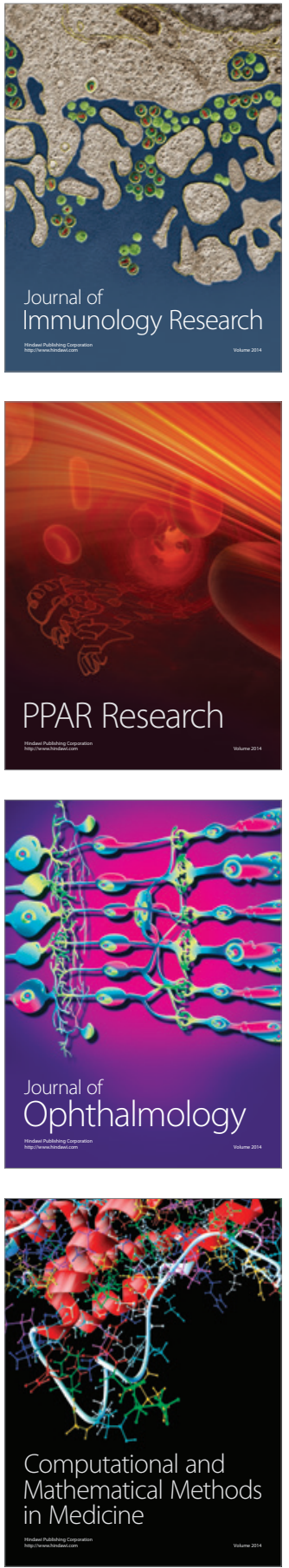

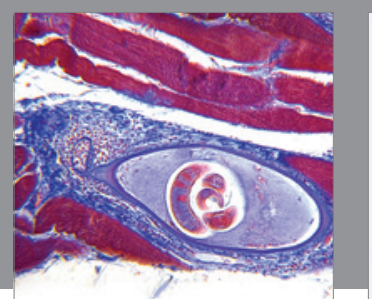

Gastroenterology Research and Practice

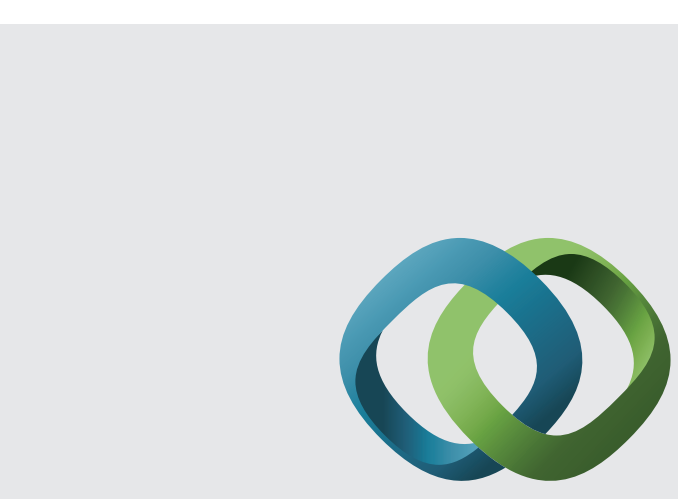

\section{Hindawi}

Submit your manuscripts at

http://www.hindawi.com
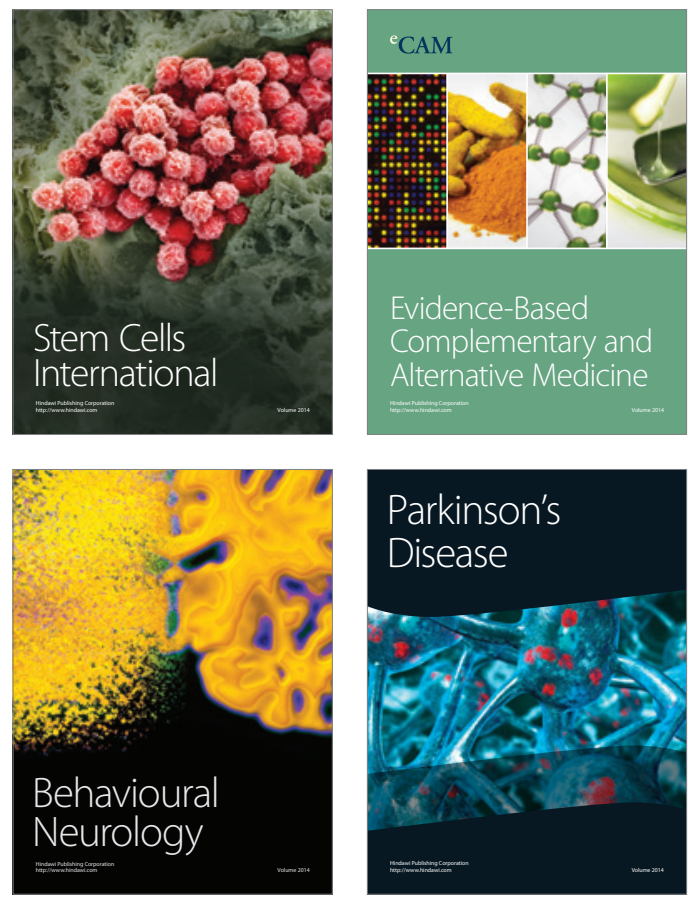
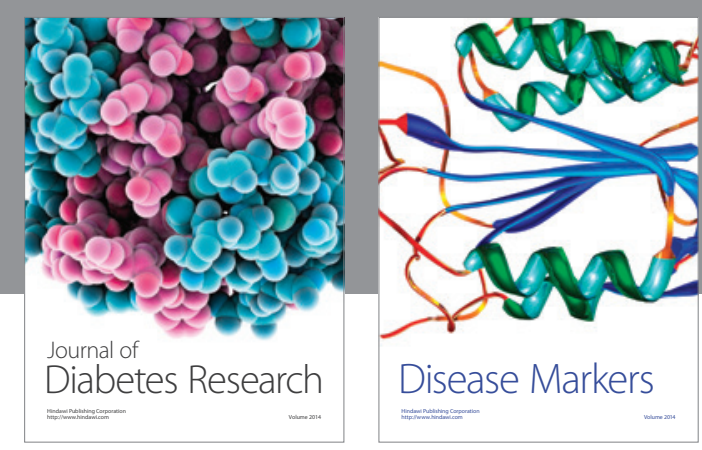

Disease Markers
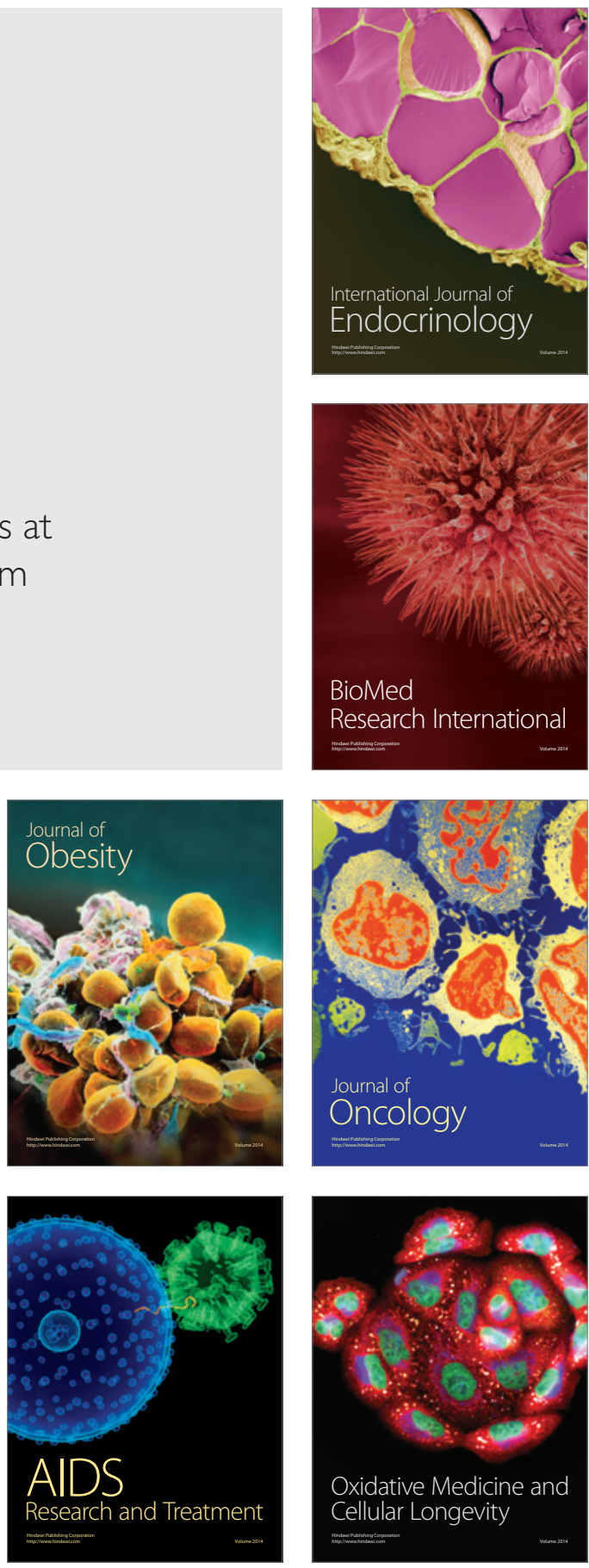